\title{
SIEMBRA CON LABRANZA TRADICIONAL Y CERO LABRANZA, MEDIANTE LA ADAPTACIÓN DE UNA SEMBRADORA DE CEREALES Y DOS DISTANCIAS ENTRE HILERAS EN CULTIVARES DE FREJOL PARA VERDE Y SECO
}

\author{
SOWING WITH TRADITIONAL AND ZERO TILLAGE BY MEANS \\ OF AN ADAPTED CEREAL SEEDER AND TWO ROW SPACINGS \\ IN GREEN AND DRY BEAN CULTIVARS
}

\author{
A. Luchsinger ${ }^{1}$; R. Villa ${ }^{2}$; G. Ocqueteau ${ }^{3} ;$ F. Suter ${ }^{3}$
}

\begin{abstract}
RESUMEN
El objetivo de esta investigación fue la comparación del desarrollo y rendimiento de los cultivares Apolo-Inia (para verde) y Torcaza-Inia (para seco), bajo dos sistemas de labranza del suelo (tradicional y cero) y dos distancias entre hileras $(52,5$ y $70,0 \mathrm{~cm})$. El estudio se realizó en la Estación Experimental Agronómica Antumapu, de la Facultad de Ciencias Agronómicas de la Universidad de Chile, ubicada en la Comuna de La Pintana, Área Metropolitana, en la temporada 1996/97.

Se utilizó una sembradora de dosificador forzado de chorro continuo, con 17 abresurcos separados a 17,5 cm. entre sí. Se sacó la barra agitadora de la tolva, cerrando las bajadas que no se emplearían. Se efectuó una prueba estática para determinar la dosis de kilos de semilla.

En una barra portaherramientas se montó un arado cincel con cuatro puntas, en cuyos extremos iba un escardillo alado. El centro de tiro de la barra se hizo calzar con el centro de tiro de la máquina para hacer coincidir las pasadas de las puntas del arado cincel con los discos sembradores a utilizar y efectuar así la labor de siembra en una sola pasada, a $7 \mathrm{~cm}$ de profundidad y $6 \mathrm{~cm}$ de ancho, simulando una sembradora de cero labranza. No hubo problema en la adaptación.

El rendimiento por hectárea fue significativamente mayor en las parcelas de labranza tradicional en ambos cultivares. Al comparar las distancias, no se observaron diferencias en frejol Apolo, pero sí en el cultivar Torcaza, para la menor distancia. Los distintos tratamientos no produjeron diferencias en la emergencia a los 8 y 16 días, y en los días de siembra a floración, en ambos cultivares. Hubo influencia en el establecimiento en frejol Torcaza en el sistema de labranza a favor de la tradicional.

La producción de vainas fue significativamente mayor en la labranza tradicional en frejol Apolo, al igual que a 70,0 cm. En el caso de Torcaza, vainas por planta, granos por vaina y peso de 100 granos no fueron afectados por el sistema de labranza. El componente más afectado por las diferentes densidades fue el número de vainas por hectárea en frejol Torcaza. El menor rendimiento individual a la mayor densidad se ve compensado por el aumento de rendimiento por unidad de superficie, siendo este aumento significativo para las densidades evaluadas.

Sería conveniente sembrar a 70,0 cm. en frejol para verde y a 52,5 en frejol para grano seco.
\end{abstract}

Palabras clave: Labranza tradicional, cero labranza, sembradora de cereales.

\begin{abstract} dosage of seed kilograms.

Parte de 2 Memorias

Facultad de Ciencias Agronómicas Universidad de Chile, Casilla 1004 Stgo.

2 Facultad de Ciencias Agronómicas Universidad de Chile, Casilla 1004 Stgo.

3 Actividad privada.
\end{abstract}

The objective of this research was to compare the development and yield of two bean cultivars, Apolo-Inia and Torcaza-Inia, for green and dry consumption, respectively, under traditional and zero tillage and two row spacings (52.5 and $70.0 \mathrm{~cm})$. Observations were carried out at the Antumapu Agricultural Experiment Station of the University of Chile, at La Pintana locality, Metropolitan Region. A seeder with a forced-dosing device of continuous flow with 17 furrow-openers spaced a $17.5 \mathrm{~cm}$ from each other was used. The shaking bar of the hopper was removed, closing the tubes that would not be used. A static test was carried out to determine the

A 4-point chisel plow was mounted on a toolbar. The drawing center of the toolbar was set to coincide with the drawing center of the machine so as the passings of the chisel plow points would coincide with the seeding disks and thus perform

Fecha de Recepción: 15 Mayo 2006

Fecha de Aceptación: 22 Junio 2006 
seeding in just one passing at $7 \mathrm{~cm}$ deep and $6 \mathrm{~cm}$ width, simulating a zero tillage seeder. No adaptations problems were observed.

Yield per hectare was significantly greater in traditional tillage plots in both cultivars. In comparing spacings, no differences were observed in the Apolo bean, but they did occur in the Torcaza cv. for the shortest distance. The different treatment showed no differences at emergences at 8 and 16 days, and from seeding to bloom in both cultivars. There was some influence on the establishment of the Torcaza bean with traditional tillage.

Pod production was significantly greater in traditional tillage in the Apolo bean as well as at $70.0 \mathrm{~cm}$ spacing. In Torcaza cv., pods per plant, grains per pod and weight of 100 grains were not affected by the tillage system. The component most affected by the different seeding rates was the number of pods per hectare in the Torcaza $c v$. The smaller individual yield at the greatest seeding rate was counterbalanced by the increased yield per unit area, this increase being significant for the evaluated seeding rates. It would be convenient to sow at a $70.0 \mathrm{~cm}$ spacing in green beans and at $52.5 \mathrm{~cm}$ in dry beans.

Key words: Traditional and zero tillage, cereal seeder.

\section{INTRODUCCIÓN}

El frejol es una fuente tradicional de alimentos y una alternativa de generación de ingresos, especialmente para el pequeño y mediano agricultor. Se cultiva para consumo en vaina verde, grano fresco (granado) y grano seco.

La superficie de frejol para verde es de aproximadamente 5.500 ha, destacándose las regiones $\mathrm{V}$ y Metropolitana y para grano seco 24.000 ha, en todo el país, en la actualidad.

El cultivo del frejol para verde ha sido destinado al consumo fresco; últimamente se ha registrado un aumento en la demanda de vainas verdes por parte de la agroindustria. Es un reemplazo de los cereales y/o como segunda siembra después de éstos. Por otro lado, el frejol para grano seco es un producto de consumo interno como generador de divisas.

Los factores de manejo para asegurar una buena producción son aquellos que tiene relación con el establecimiento del cultivo, destacándose época de siembra, dosis de semilla, forma de siembra, densidad de plantas y calidad de la semilla.

La labranza conservacionista del suelo constituye un camino, entendiéndose por ella todo lo que implique una máxima retención de residuos sobre la superficie del suelo (Crovetto, 1992). Esto involucra cambios en el ambiente edáfico y en el manejo del suelo, suprimiendo la preparación del mismo, usándose maquinaria especial y los herbicidas apropiados (Luchsinger et al., 1979).

La labranza tradicional se caracteriza por el laboreo del suelo y procesamientos del rastrojo.

El agricultor tradicional de la zona central dispone de una máquina sembradora de chorrillos, que puede usarse previas adaptaciones para siembra mecánica del frejol. Esto debe considerarse al plantear un uso alternativo del suelo, ya que nuevas inversiones están muy limitadas para ellos, recomendándose hacer cambios graduales.

Es importante ensayar manejos que permitan un mayor rendimiento en el cultivo del frejol bajo diferentes distancias de siembra, donde la densidad poblacional pasa a ser un factor de gran interés y que depende de la variedad a sembrar.

La sembradora de chorro continuo está diseñada para sembrar cereales y forrajes en hileras espaciadas entre 15 y $20 \mathrm{~cm}$; para sembrar otros cultivos en hileras se eliminan bajadas.

La sembradora de cero labranza realiza todas las funciones de una sembradora, sin embargo, prepara sólo una franja de unos $5 \mathrm{~cm}$ de ancho por 8-10 de profundidad.

Ensayos efectuados por los dos autores principales indican que el realizar adaptaciones de una máquina sembradora de chorrillos con arado cincel para hacer cero labranza, no presentó problemas en su ejecución y las pruebas de terreno proporcionaron resultados satisfactorios en cuanto a rendimiento, emergencia de plantas y control de malezas.

En el cultivar Apolo, las distintas densidades inciden directamente sobre el número de vainas, no importando el número de granos por vaina y el peso de los 100 granos; en Torcaza, influyen estos últimos.

En frejol Apolo (vainas verdes), lo normal es obtener entre 60 y 70 qq, pudiéndose lograr hasta 100 o más. En frejol para grano seco, Torcaza, los rendimientos son variables, lográndose 30 qq o más por ha (promedio nacional:13,3 qq).

El objetivo es la comparación del desarrollo y rendimiento de los cultivares de frejol Apolo-INIA (para verde) y Torcaza INIA (para seco) bajo dos sistemas de labranza del suelo (tradicional y cero) $\mathrm{y}$ en dos distancias de siembra $(52,5$ y $70,0 \mathrm{~cm})$. 


\section{MATERIALES Y MÉTODOS}

La investigación se realizó en la Estación Experimental Agronómica Antumapu, de la Facultad de Ciencias Agronómicas de la Universidad de Chile, ubicada en la Comuna de La Pintana, Área Metropolitana.

Se utilizó un diseño factorial de $2 \times 2$ en bloques completamente al azar con cuatro repeticiones. Las parcelas fueron de cuatro hileras y de $10 \mathrm{~m}$ de longitud, separadas a 52,5 y $70,0 \mathrm{~cm}$, respectivamente. Se usaron dos sistemas de labranza (tradicional y cero).

La labranza tradicional consistió en: riegoaradura-dos rastrajes-siembra. Control químico y manual de maleza. En cero labranza: riego-control químico de malezas-siembra. Control posterior químico y manual.

Se utilizó una sembradora de dosificador forzado de chorro continuo, con 17 abresurcos separados a 17,5 cm entre sí. Se sacó la barra agitadora de la tolva, cerrando las bajadas que no se emplearían. Se efectuó una prueba estática para determinar la dosis de kilos de semillas.

En una barra portaherramientas se montó un arado cincel con cuatro puntas, en cuyos extremos iba un escardillo alado. El centro de tiro de la barra se hizo calzar con el centro de tiro de la máquina para hacer coincidir las pasadas de las puntas del arado cincel con los discos sembradores a utilizar y efectuar así la labor de siembra en una sola pasada, a $7 \mathrm{~cm}$ de profundidad y $6 \mathrm{~cm}$ de ancho, simulando una sembradora de cero labranza.

Para el control de malezas, en labranza tradicional se utilizó Dual (1,5 1 del producto comercial/ha), de presiembra incorporado con rastraje, en ambos cultivares. En cero labranza, para el cultivar Apolo se usó Gramoxone Super (Paraquat), en dosis de 2,0 1 de producto comercial, como desecante, cinco días después de la siembra. En el cultivar Torcaza se empleó Alanex 48 EC, en la preemergencia del cultivo, en dosis de 41 por ha. Con el cultivo emergido (dos hojas trifoliadas), se aplicó Basagran (1,5 1 de producto comercial), en ambos cultivares y sistemas de labranza, para el control de malezas de hoja ancha y gramíneas anuales y, además, en Torcaza se aplicó Assureplus para malezas perennes en ambas labranzas. Limpias manuales.

Se aplicaron $60 \mathrm{u}$. de fósforo por hectárea en la siembra en ambos frejoles; 40 de nitrógeno, en dos parcialidades, $1 / 3$ a la siembra y el resto antes de floración para Torcaza y 60 para Apolo, 1/2 a la siembra y el resto antes de floración.

Todas las mediciones se efectuaron entre el tercer y sexto metro de las dos hileras centrales de cada parcela.

Ellas fueron:

- Profundidad de siembra (cm). Después de la siembra.

- Emergencia ( $\mathrm{N}^{\circ}$ plantas/m lineal), a los 8 y 16 días de la siembra.

- Establecimiento ( $\mathrm{N}^{\circ}$ de plantas/m lineal), a los 30 días de la siembra y a la cosecha en Torcaza y a la siembra y primera cosecha en el frejol Apolo.

- Producción de vainas $(\mathrm{N} \% \mathrm{~m}$ lineal y $\mathrm{N} \%$ plantas). Se suma el número de vainas de los dos primeros cortes.

- Peso de vainas por $m$ lineal (g). Se pesó la de los dos primeros cortes (Apolo).

- Grano por vaina y peso de 100 granos (Torcaza).

- Rendimiento (qq/ha de vainas y grano).

En relación a la cosecha, en frejol para verde se efectuaron tres cortes de vainas: a los 65,70 y 76 días de la siembra, cuando la mayoría de las vainas alcanzó su tamaño máximo, sin granos en formación. Para el análisis estadístico se contabilizaron sólo los dos primeros cortes.

Para grano, la cosecha se realizó cuando las plantas y vainas presentaron amarillez casi completa, ocurriendo a los 101 días de la siembra.

\section{RESULTADOS Y DISCUSIÓN}

\section{PROFUNDIDAD DE SIEMBRA}

La profundidad de siembra es esencial para asegurar una población óptima de plantas por hectárea. Dado que se utilizó la misma sembradora en ambos sistemas de labranza y distancias, las diferencias en la uniformidad de la profundidad de siembra tienen su origen en el distinto manejo del suelo. A pesar de ello, la profundidad de siembra fue bastante pareja en ambos cultivares, variando entre 8,0 y $7,5 \mathrm{~cm}$ para la tradicional y cero, respectivamente. En distancias de siembra, los valores fueron 7,6 y $7,9 \mathrm{~cm}$, para 70,0 y 52,5 $\mathrm{cm}$, respectivamente.

Concuerda con lo expresado por Figueroa (1996). 


\section{EMERGENCIA}

En relación a la emergencia a los 8 y 16 días, por metro lineal, los datos se presentan en el Cuadro 1 y podemos observar que no hay diferencias significativas en los tratamientos de labranza, en ambas mediciones y cultivares, pero sí hay una mayor población a los 16 días. Sin embargo, la emergencia es ligeramente inferior en los tratamientos de cero labranza, lo que puede tener su origen en el microrrelieve. En distancias entre hileras no existieron diferencias en ambas mediciones, eso sí se observó un aumento de plantas emergidas a los 16 días, producto del primer riego, que se efectuó 12 días de la siembra, riesgo que es frecuente y que afecta en especial a la maduración de la vainas para consumo verde. Cabe hacer presente que en el frejol Torcaza hubo mayor población a la menor distancia.

Es importante el número de plantas por metro lineal ya que nos indica la distancia a la cual quedaron las plantas. Para Apolo, las distancias variaron entre 7,3 y 7,7 cm a los 8 días, bajando a 6,4 y $6,9 \mathrm{~cm}$ a los 16 días. En Torcaza, fue entre 9,9 y $11,8 \mathrm{~cm}$ a los 8 días y entre 7,6 y 8,5 a los 16 días. En todos los casos las distancias se consideran adecuadas.

La evaluación del efecto de las densidades sobre la emergencia del cultivo se aprecia mejor al exponer los resultados en base a una unidad de superficie (metro cuadrado), los que se presentan en el Cuadro 2. El efecto de la mayor dosis de semilla por hectárea se observa tanto a los 8 como a los 16 días después de la siembra, siendo significativo en el frejol Torcaza y a los 16 días en Apolo.

Cuadro 1

Plantas emergidas en frejol Apolo y Torcaza (metro lineal)

\begin{tabular}{|l|c|c|c|c|}
\cline { 2 - 5 } \multicolumn{1}{c|}{} & \multicolumn{2}{c|}{ 8 días } & \multicolumn{2}{c|}{ 16 días } \\
\cline { 2 - 5 } & Apolo & Torcaza & Apolo & Torcaza \\
\hline Labranza tradicional & $13,6 \mathrm{a}$ & $10,2 \mathrm{a}$ & $15,7 \mathrm{a}$ & $13,0 \mathrm{a}^{*}$ \\
Cero labranza & $12,9 \mathrm{a}$ & $8,5 \mathrm{a}$ & $14,6 \mathrm{a}$ & $12,0 \mathrm{a}$ \\
\hline Distancia entre hileras (cm) & & & & $11,8 \mathrm{a}$ \\
70,0 & $13,4 \mathrm{a}$ & $8,9 \mathrm{a}$ & $15,3 \mathrm{a}$ & $13,2 \mathrm{a}$ \\
52,5 & $13,1 \mathrm{a}$ & $9,8 \mathrm{a}$ & $15,0 \mathrm{a}$ & \\
\hline
\end{tabular}

(*) Letras distintas indican significación.

Cuadro 2

Plantas emergidas en frejol Apolo y Torcaza $\left(\mathbf{m}^{2}\right)$

\begin{tabular}{|l|c|c|c|c|}
\cline { 2 - 5 } \multicolumn{1}{c|}{} & \multicolumn{2}{c|}{8 días } & 16 días \\
\cline { 2 - 5 } & Apolo & Torcaza & $25,6 \mathrm{a}$ & Torcaza \\
\hline Labranza tradicional & $22,1 \mathrm{a}$ & $16,6 \mathrm{a}$ & $21,3 \mathrm{a}^{*}$ \\
Cero labranza & $21,1 \mathrm{a}$ & $13,9 \mathrm{a}$ & & $19,5 \mathrm{a}$ \\
\hline Distancia entre hileras (cm) & & & $21,8 \mathrm{a}$ & $16,9 \mathrm{a}$ \\
70,0 & $19,1 \mathrm{a}$ & 12,7 & $28,5 \mathrm{~b}$ & $25,1 \mathrm{~b}$ \\
52,5 & $24,9 \mathrm{a}$ & $18,6 \mathrm{~b}$ & & \\
\hline
\end{tabular}

(*) Letras distintas indican significación. 
Distinta es la situación que ocurre comparando los dos sistemas de labranza, ya que las diferencias en la emergencia no son significativas, pero hay mayor número de plantas en la labranza tradicional. Sin embargo, las diferencias proyectadas a una hectárea producen pérdidas que varían entre 18.600 y 17.600 plantas para Apolo y Torcaza, respectivamente, si consideramos la población a los 16 días, en detrimento de la cero labranza.

\section{ESTABLECIMIENTO}

$\mathrm{Al}$ evaluar las plantas establecidas a los 30 días y a la cosecha, por metro cuadrado (Cuadro 3 ), se observa que los resultados a través de los sistemas de labranza no presentan diferencias en el cultivar Apolo, pero sí una mayor cantidad en labranza tradicional. Torcaza presentó diferencias significativas entre las labranzas en ambos parámetros, siempre a favor de la tradicional. Al comparar las poblaciones a los 30 días con aquellas producidas a los 16, puede señalarse que ellas bajaron en Apolo y aumentaron en Torcaza.

$\mathrm{Al}$ analizar las distancias entre hileras, se encontraron diferencias entre ellas, tanto a los 30 días como a la cosecha. Si comparamos la población de los 30 días con las encontradas a los 16, podemos notar que ellas disminuyeron levemente en Apolo, observándose un número superior en la menor distancia.

El número de plantas por metro cuadrado es significativamente superior en la distancia de 52,5 $\mathrm{cm}$ entre hileras, en ambos cultivares y mediciones. Por otro lado, las poblaciones a los 30 días y a la cosecha no muestran diferencias, siendo las mismas en Apolo y levemente inferiores en Torcaza.

\section{PRODUCCIÓN DE VAINAS}

Al comparar las labranzas (metro lineal), presentadas en el Cuadro 4, se observaron diferencias significativas a favor de la tradicional en el número de vainas para Apolo $(35,2 \%)$. Como el número de plantas establecidas a los 30 días y al momento de la primera cosecha son iguales, las diferencias deben explicarse por un mayor desarrollo de las plantas en la labranza tradicional, produciendo, en promedio, más vainas. La literatura indica que se requieren 3-4 años antes que los suelos, bajo mínima labranza, desarrollen una porosidad más favorable.

En frejol Torcaza, si bien hubo una diferencia a favor de la labranza tradicional (21,5\%), ésta no fue significativa. Sin embargo, a diferencia del cultivar Apolo, aquí hubo significación a favor de la labranza tradicional en las plantas establecidas a los 30 días y a la cosecha, lo que explicaría la diferencia.

En relación a las distancias entre hileras, se observó diferencia significativa a favor de la distancia mayor en frejol Apolo, con 34,9\%. El primer corte rindió un 61,5\% y el segundo 38,5 (suma de los dos). El tercero produjo un $14,3 \%$ del total. Como el número de plantas establecidas es el mismo en las distancias de siembra, la diferencia se atribuye a la menor competencia a que están sujetas las plantas en la distancia mayor. Para el frejol Torcaza no hubo diferencias por efecto de la distancia, pero se observó un aumento de $10,3 \%$ a favor de la mayor.

Cuadro 3

Plantas establecidas en frejol Apolo y Torcaza $\left(\mathbf{m}^{2}\right)$

\begin{tabular}{|l|c|c|c|c|}
\cline { 2 - 5 } \multicolumn{1}{c|}{} & \multicolumn{2}{c|}{ A los 30 días } & \multicolumn{2}{c|}{ A la cosecha } \\
\cline { 2 - 5 } \multicolumn{1}{c|}{} & Apolo & Torcaza & Apolo & Torcaza \\
\hline Labranza tradicional & $24,0 \mathrm{a}$ & $23,6 \mathrm{a}$ & $24,0 \mathrm{a}$ & $23,2 \mathrm{a} *$ \\
Cero labranza & $23,4 \mathrm{a}$ & $21,0 \mathrm{~b}$ & $23,4 \mathrm{a}$ & $20,4 \mathrm{~b}$ \\
\hline Distancia entre hileras (cm) & & & & $18,1 \mathrm{~b}$ \\
70,0 & $20,5 \mathrm{~b}$ & $18,9 \mathrm{~b}$ & $20,5 \mathrm{~b}$ & $25,5 \mathrm{a}$ \\
52,5 & $27,8 \mathrm{a}$ & $26,9 \mathrm{a}$ & $27,8 \mathrm{a}$ & \\
\hline
\end{tabular}

(*) Letras distintas indican significación. 
Cuadro 4

Producción de vainas en frejol Apolo y Torcaza

\begin{tabular}{|l|c|c|c|c|}
\cline { 2 - 5 } \multicolumn{1}{c|}{} & \multicolumn{4}{c|}{ Producción de vainas } \\
\cline { 2 - 5 } & \multicolumn{2}{c|}{ Metro lineal } & \multicolumn{2}{c|}{ Metro cuadrado } \\
\cline { 2 - 5 } & Apolo & Torcaza & $228,5 \mathrm{a}$ & $198,5 \mathrm{a}^{*}$ \\
\hline Labranza tradicional & $139,9 \mathrm{a}$ & $121,6 \mathrm{a}$ & $169,0 \mathrm{~b}$ & $163,4 \mathrm{a}$ \\
Cero labranza & $103,5 \mathrm{~b}$ & $100,1 \mathrm{a}$ & & \\
\hline Distancia entre hileras (cm) & & & $199,8 \mathrm{a}$ & $163,8 \mathrm{a}$ \\
70,0 & $139,8 \mathrm{a}$ & $114,7 \mathrm{a}$ & $197,4 \mathrm{a}$ & $198,1 \mathrm{a}$ \\
\hline 52,5 & $103,6 \mathrm{~b}$ & $104,0 \mathrm{a}$ & & \multicolumn{2}{c}{ Torcaza } \\
\hline
\end{tabular}

(*) Letras distintas indican significación.

Si consideramos la producción de vainas por metro cuadrado (Cuadro 4), que es más importante, se observa en las labranzas una mayor producción en la tradicional, significativa, que alcanza a un 35,2\% en frejol Apolo. En Torcaza existe una diferencia de $21,5 \%$ a favor de la tradicional, pero no significativa. En los valores para distancia entre hileras no se presentaron diferencias en ningún cultivar. Sin embargo, en Apolo la distancia mayor produjo 1,2\% más; en Torcaza, la distancia menor logró un $20,9 \%$ más, producto de la mayor cantidad de plantas establecidas ya que frejol para grano seco se cosecha al final del período vegetativo.

\section{PESO DE VAINAS}

Esta variable se midió sólo en frejol Apolo (Cuadro 5), por ser de gran importancia en el cultivo para verde. Si tomamos en cuenta por metro lineal la labranza tradicional y la distancia de $70 \mathrm{~cm}$ fueron superiores en un 38,4 y $39,2 \%$, respectivamente. $\mathrm{Al}$ considerar el peso por metro cuadrado, la labranza tradicional fue diferente significativamente de la cero en un $38,4 \%$, mayor peso que se debe al número de vainas observadas. En la labranza tradicional el primer corte rindió un 59,5\% y el segundo 40,5; en cero labranza, el $65,3 \%$ lo produjo el primero y el 34,7 el segundo (suma de los dos). Un tercer corte produjo 8,7 y $11,4 \%$ en la tradicional y cero. Al comparar el peso de vainas en relación a las distancias entre hileras, la mayor distancia produjo un $39,2 \%$ más que $52,5 \mathrm{~cm}$. En la distancia mayor, el primer corte aportó el 59,4\% y el segundo un 40,6; el tercero logró un 8,4\%. En la distancia menor, el primer y segundo corte aportaron el 65,3 y $34,7 \%$, respectivamente. Al efectuar un tercer corte, este aportó un 11,8\% del total. La diferencia a favor de la distancia mayor fue de solo un 3,9\%, o sea, hubo una compensación.

\section{GRANOS POR VAINA Y PESO DE 100 GRANOS}

Los valores se presentan en el Cuadro 6, sólo para el cultivar Torcaza, ya que ellos influyen directamente en el rendimiento final.

Estos caracteres demostraron un alto grado de independencia con respecto a los sistemas de labranza y distancia entre hileras, siendo muy parecidos (Cuadro 6).

Si consideramos el número de granos por metro cuadrado observamos diferencias favorables a la labranza tradicional en un $19,6 \%$, no siendo significativa; en las distancias la diferencia a favor de la menor alcanzo a $19,4 \%$, debido a la mayor cantidad de plantas por superficie.

En el caso del peso de 100 granos, lo único por comentar es la diferencia de 3 gramos a favor de la labranza tradicional; en las distancias, la diferencia alcanza a 0,7 g., en beneficio de la menor. Estas diferencias se reflejan en el rendimiento final.

\section{RENDIMIENTO}

Los resultados se presentan en el Cuadro 7, pudiendo observarse diferencias significativas entre los sistemas de labranza a favor de la tradicional 
Cuadro 5

Peso de vainas en frejol Apolo

\begin{tabular}{|l|c|c|}
\cline { 2 - 3 } \multicolumn{1}{c|}{} & \multicolumn{2}{c|}{ Peso de vainas } \\
\cline { 2 - 3 } \multicolumn{1}{c|}{} & Metro lineal & Metro cuadrado \\
\hline Labranza tradicional & $854,6 \mathrm{a}$ & $1.395,3 \mathrm{a}^{*}$ \\
Cero labranza & $617,5 \mathrm{~b}$ & $1.008,2 \mathrm{~b}$ \\
\hline Distancia entre hileras (cm) & & \\
70,0 & $859,4 \mathrm{a}$ & $1.221,2 \mathrm{a}$ \\
52,5 & $617,3 \mathrm{a}$ & $1.175,7 \mathrm{a}$ \\
\hline
\end{tabular}

(*) Letras distintas indican significación.

Cuadro 6

Granos por vainas y por metro cuadrado y peso de 100 granos en frejol Torcaza

\begin{tabular}{|c|c|c|c|}
\hline & \multicolumn{2}{|c|}{ Número de granos } & \multirow{2}{*}{ Peso de 100 granos } \\
\hline & Por vaina & Por metro cuadrado & \\
\hline Labranza tradicional & $3,9 \mathrm{a}$ & $761,7 \mathrm{a}$ & $40,9 a^{*}$ \\
\hline Cero labranza & $3,9 \mathrm{a}$ & $636,8 \mathrm{a}$ & $38,0 \mathrm{a}$ \\
\hline \multicolumn{4}{|l|}{ Distancia entre hileras $(\mathrm{cm})$} \\
\hline 70,0 & $3,9 \mathrm{a}$ & $637,4 a$ & $39,1 \mathrm{a}$ \\
\hline 52,5 & $3,9 \mathrm{a}$ & $761,1 \mathrm{a}$ & $39,8 \mathrm{a}$ \\
\hline
\end{tabular}

(*) Letras distintas indican significación.

en ambos cultivares. Los rendimientos coinciden con Figueroa (1996).

Al analizar el caso de Apolo, la diferencia alcanza a 38,1 qq y ella se debe a que las parcelas de cero labranza se vieron afectadas por el riego utilizado y a la compactación del suelo y a un menor peso de ellas.

El haber obtenido mayor rendimiento en la labranza tradicional no implica mayor rentabilidad, ya que para ello sería necesario un análisis de costo/beneficio, puesto que los costos son diferentes, siendo más bajos en cero labranza. En labranza tradicional, el primer corte produjo un 59,7\% y el segundo un 40,3; para cero labranza, los porcentajes fueron 66,0 y $34,0 \%$ respectivamente. El tercer corte en ambos sistemas fue muy bajo. Esto indica que el primer corte es el más importante y el de mejor calidad de vainas.

En frejol Torcaza, la diferencia alcanzó a 7 qq. El menor rendimiento de las parcelas con cero la- branza se debería a la incidencia del microrrelieve, el que afectó la emergencia y establecimiento de plantas. Los componentes de rendimiento no fueron afectados significativamente por la labranza.

Al comparar las distancias entre hileras, en frejol Apolo no existió significación, obteniéndose sólo 4,9 qq a favor de la mayor distancia. La mayor densidad poblacional compensa el menor rendimiento individual de cada planta. Para el cultivar Torcaza, sí hubo significación, siendo la diferencia de 5,4 quintales a favor de la distancia menor. Esto se explicaría por el hecho de que la cosecha se realiza cuando la planta está seca y se cosechan todas las vainas. Con ello se reafirma el hecho de que el cultivar en referencia responde positivamente a mayores densidades poblacionales. Hay menor rendimiento individual por planta a mayores densidades, pero aumenta el rendimiento por unidad de superficie, que compensa dicho efecto. 
Cuadro 7

Rendimiento en frejol Apolo y Torcaza

\begin{tabular}{|l|c|c|c|c|c|}
\cline { 2 - 6 } & \multicolumn{4}{c|}{ Rendimiento (qq/ha) } \\
\cline { 2 - 6 } & \multicolumn{3}{|c|}{ Apolo } & \multicolumn{2}{c|}{ Torcaza } \\
\cline { 2 - 6 } & $\mathbf{1}^{\text {er }}$ corte & $\mathbf{2}^{\text {do }}$ corte & Total & 3 $^{\text {er }}$ corte & Total \\
\hline Labranza tradicional & 82,8 & 56,0 & $138,8 \mathrm{a}$ & 13,5 & $31,0 \mathrm{a}^{*}$ \\
Cero labranza & 66,4 & 34,3 & $100,7 \mathrm{~b}$ & 13,3 & $24,0 \mathrm{~b}$ \\
\hline Distancia entre hileras (cm) & & & & & \\
70,0 & 72,6 & 49,6 & $122,2 \mathrm{a}$ & 11,1 & $24,8 \mathrm{a}$ \\
52,5 & 76,6 & 40,7 & $117,3 \mathrm{a}$ & 15,6 & $30,2 \mathrm{~b}$ \\
\hline
\end{tabular}

(*) Letras distintas indican significación.

Con respecto al rendimiento obtenido en frejol Apolo, se observa que éste fue superior a 100 qq en todos los casos, resultando conveniente analizarlo a la luz de otras experiencias, debido a que la siembra se efectuó en una época considerada tardía (mediados de diciembre), y la floración se produjo con altas temperaturas. Figueroa (1996), quien lo sembró a fines de noviembre, obtuvo un rendimiento superior a 100 qq. Sepúlveda (1983), a mediados de diciembre, logró 130 qq. De acuerdo a estos antecedentes, el rendimiento logrado en este ensayo estaría dentro del rango comúnmente obtenido para esta fecha de siembra en la zona central.

\section{CONCLUSIONES}

El rendimiento por hectárea fue significativamente mayor en las parcelas de labranza tradicional en ambos cultivares. Al comparar las distancias, no se observaron diferencias en frejol Apolo, pero sí en el cultivar Torcaza, para la menor distancia.

\section{LITERATURA CITADA}

CROVETTO, C. Rastrojo sobre el suelo: una introducción a la cero labranza. Edit. Universitaria. Chile 301 pág. 1992.

FIGUEROA, A. "Comparación de diferentes sistemas dosificadores de siembras mecanizadas de fréjol cultivar Apolo bajo dos distancias entre hileras. Tesis Ing. Agr. Universidad de Chile, Facultad de Ciencias Agronómicas y Forestales. 65 pág. 1996.

LUCHSINGER, A.; VILLA, R.; GAETE, M. Efecto de la labranza mínima y siembra sin labor en el desarrollo
Utilizar una máquina sembradora de chorro continuo y simular una de cero labranza, no presentó dificultades técnicas ni demandó una gran inversión.

Los distintos tratamientos no produjeron diferencias en la emergencia a los 8 y 16 días, como tampoco en los días de siembra a floración en ambos cultivares. Hubo influencia en el establecimiento en frejol Torcaza en el sistema de labranza, a favor de la tradicional.

La producción de vainas fue significativamente mayor en la labranza tradicional en frejol Apolo, al igual que a 70,0 cm. En el caso de Torcaza, vainas por planta, granos por vaina y peso de 100 granos no fueron afectados por el sistema de labranza.

De los componentes de rendimiento, el más afectado por las diferentes densidades fue el número de vainas por hectárea en frejol Torcaza. El menor rendimiento individual a la mayor densidad se ve compensado por el aumento de rendimiento por unidad de superficie, siendo este aumento significativo para las densidades evaluadas.

Sería conveniente sembrar a 70,0 cm en frejol para verde y a 52,5 en frejol para grano seco.

y productividad del maíz (Zea mays L.) Inv. Agrícola, Vol. 5(1):39-45. 1979.

LUCHSINGER, A.; VILLA, R.; OSSANDÓN, E.; FRITSCH, N.; VILLALOBOS, P. "Adaptación de equipos convencionales para siembra de maíz con labranza cero y su comparación con otros métodos. Inv. Agrícola, Vol. 11 (1-2): 47-58. 1991.

SEPÚLVEDA, H. "Producción de frejoles para verde y granado". El campesino. Vol. 114. Soc. Nac. de Agricultura. 1983. 48 pág. 